\title{
Potential for Malignancy
}

National Cancer Institute

\section{Source}

National Cancer Institute. Potential for Malignancy. NCI Thesaurus. Code C160575.

Relating to the potential of a neoplasm to undergo malignant transformation. 